\title{
A COMPARISON THEOREM FOR OPERATORS WITH COMPACT RESOLVENT
}

\author{
REESE T. PROSSER
}

ABstract. The asymptotic behavior of the eigenvalue sequence of a semibounded operator with compact resolvent is stable under relatively bounded perturbations.

It is sometimes useful to have available results providing a means of comparing the "size" of a given operator with that of another [5]. This is particularly true when their difference is "small," so that one of them can be regarded as a perturbation of the other. We present here an elementary result of this nature, valid for operators with compact resolvent acting on a Hilbert space, which derives from the work of Erhard Heinz [4]. We obtain from this result that the asymptotic behavior of the eigenvalue sequence of such an operator is stable under relatively bounded perturbations, thus providing a modest improvement on a recent result of Richard Beals [1].

Let $H$ be a Hilbert space and $A$ a closed nonnegative operator with domain $\operatorname{dom}(A)$ dense in $H$. Denote by $A^{1 / 2}$ the unique closed nonnegative square root of $A$.

Let $B$ be another such operator, and suppose

(a) $\operatorname{dom}\left(A^{1 / 2}\right) \supset \operatorname{dom}\left(B^{1 / 2}\right)$, and

(b) $\left\|A^{1 / 2} x\right\| \leqq\left\|B^{1 / 2} x\right\|$ for all $x \in \operatorname{dom}\left(B^{1 / 2}\right)$.

Then we say $B$ majorizes $A$, and write $A \leqq B$. If $B$ is bounded and majorizes $A$, then clearly $A$ is bounded, and $(A x, x) \leqq(B x, x)$ for all $x \in H$. Moreover, if $I \leqq B$, then $B$ is invertible, and $I \geqq B^{-1}$.

For such operators we have the following sequence of results:

Lemma 1. If $\operatorname{dom}(A) \supset \operatorname{dom}(B)$, then for some positive constant $k$ we have $(I+A)^{2} \leqq k^{2}(I+B)^{2}$.

Proof. Define on $\operatorname{dom}(A)$ and $\operatorname{dom}(B)$ the norms $\|x\|_{A}=\|(I+A) x\|$ and $\|x\|_{B}=\|(I+B) x\|$, respectively, and note that because $A$ and $B$ are closed, $\operatorname{dom}(A)$ and $\operatorname{dom}(B)$ become Hilbert spaces under these norms. Moreover, the injection $J: \operatorname{dom}(B) \rightarrow \operatorname{dom}(A)$ is evidently closed under these norms. By the Closed Graph Theorem, $J$ is bounded, and $\|x\|_{A}=\|J x\|_{A} \leqq\|J\|\|x\|_{B}$ for all $x \in \operatorname{dom}(B)$, as required [3].

Received by the editors April 27, 1970.

AMS 1969 subject classifications. Primary 4710, 4745, 4748.

Key words and phrases. Asymptotic behavior, eigenvalue sequence, semibounded operators, compact resolvent, relatively bounded perturbations. 
Lemma 2. If $(I+A)^{2} \leqq k^{2}(I+B)^{2}$, then $(I+A) \leqq k(I+B)$.

Lemma 3. If $(I+A) \leqq k(I+B)$, then $(I+A)^{-1} \geqq k^{-1}(I+B)^{-1}$.

The proofs of these lemmas are found in the work of Erhard Heinz [4], who provided a general framework for the study of such questions.

Now suppose that $(I+A)^{-1}$ is compact. Then we know that $A$ has a pure point spectrum, and we can arrange the eigenvalues in order of increasing magnitude: $0 \leqq a_{1} \leqq a_{2} \leqq \cdots \leqq a_{n} \uparrow \infty$. Moreover, if $(I+A)^{-1} \geqq k(I+B)^{-1}$, then $(I+B)^{-1}$ is also compact, and we can arrange the eigenvalues $b_{n}$ of $B$ similarly.

Lemma 4. If $(I+A)^{-1}$ is compact, and $(I+A)^{-1} \geqq k^{-1}(I+B)^{-1}$ then $\left(1+a_{n}\right)^{-1} \geqq k^{-1}\left(1+b_{n}\right)^{-1}$ for all $n$.

Proof. This follows from the variational definition of the eigenvalues $\left(1+a_{n}\right)^{-1}$ and $\left(1+b_{n}\right)^{-1}$ of $(I+A)^{-1}$ and $(I+B)^{-1}$, respectively [3].

Combining these results, we obtain

ThEOREM 5. If $(I+A)^{-1}$ is compact, and $\operatorname{dom}(A) \supset \operatorname{dom}(B)$, then $(I+B)^{-1}$ is compact, and for some positive constant $k$ we have $\left(1+a_{n}\right)$ $\leqq k\left(1+b_{n}\right)$ for all $n$.

Now write $V=A-B$, and suppose $V$ is bounded relative to $B$ : $\|V x\| \leqq c\|(I+B) x\|$ for some $c>0$ and all $x \in \operatorname{dom}(B)$. Then we have

$$
\|(I+A) x\| \leqq\|(I+B) x\|+\|V x\| \leqq(1+c)\|(I+B) x\| .
$$

Moreover, if $c<1$, then

$$
\|V x\| \leqq c\|(I+A-V) x\| \leqq c\|(I+A) x\|+c\|V x\|,
$$

so $(1-c)\|V x\| \leqq c\|(I+A) x\|$ and $\|V x\| \leqq c(1-c)^{-1}\|(I+A) x\|$. Hence,

$$
\|(I+B) x\| \leqq\|(I+A) x\|+\|V x\| \leqq(1-c)^{-1}\|(I+A) x\| .
$$

Under these circumstances we have

$$
(1-c)\|(I+B) x\| \leqq\|(I+A) x\| \leqq(1+c)\|(I+B) x\| .
$$

CoRollary. If in addition $\|(A-B) x\| \leqq c\|(I+B) x\|$ for all $x$ $\in \operatorname{dom}(B)$ and some constant $0<c<1$, then we have $\operatorname{dom}(A)=\operatorname{dom}(B)$, and

$$
(1-c)\left(1+a_{n}\right) \leqq\left(1+b_{n}\right) \leqq(1+c)\left(1+a_{n}\right) \quad \text { for all } n .
$$

These results extend easily to semibounded operators by replacing 
$A$ by $A+a I$ for large $a>0$, and to general operators by replacing $A$ by $\left(A A^{*}\right)^{1 / 2}[5]$.

\section{BIBLIOGRAPHY}

1. Richard Beals, On eigenvalue distributions for elliptic operators without smooth coefficients, Bull. Amer. Math. Soc. 72 (1966), 701-705. M R 33 \#4449.

2. Colin Clark, The asymptotic distribution of eigenvalues, SIAM Rev. 9 (1967), 627-646.

3. Nelson Dunford and J. T. Schwartz, Linear operators. II: Spectral theory. Selfadjoint operators in Hilbert space, Interscience, New York, 1963. MR 32 \#6181.

4. Erhard Heinz, Beiträge zur Störungstheorie der Spektralzerlegung, Math Ann. 123 (1951), 415-438. MR 13, 471.

5. Reese T. Prosser, On a similarity invariant for compact operators, Trans. Amer. Math. Soc. 134 (1968), 171-181. MR 37 \#3393.

Dartmouth College, Hanover, New Hampshire 03755 